\title{
Blastic plasmacytoid dendritic cell neoplasm frequently shows occult central nervous system involvement at diagnosis and benefits from intrathecal therapy
}

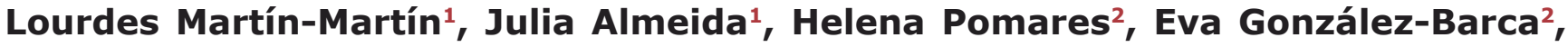 \\ Pilar Bravo ${ }^{3}$, Teresa Giménez ${ }^{4}$, Cecilia Heras ${ }^{5}$, José-Antonio Queizán 6 , Elena Pérez- \\ Ceballos $^{7}$, Violeta Martínez ${ }^{8}$, Natalia Alonso9 ${ }^{9}$ Carlota Calvo ${ }^{10}$, Rodolfo Álvarez ${ }^{11}$, \\ María Dolores Caballero ${ }^{12}$ and Alberto Orfao ${ }^{1}$ \\ ${ }^{1}$ Cancer Research Centre (IBMCC, USAL-CSIC), Institute for Biomedical Research of Salamanca (IBSAL) and Department of \\ Medicine and Cytometry Service (NUCLEUS Research Support Platform), University of Salamanca (USAL), Salamanca, Spain \\ ${ }^{2}$ Hematology Department, Institut Catalá d'Oncologia, Hospital Duran i Reynals, University of Barcelona, Institut d'Investigació \\ Biomèdica de Bellvitge, Barcelona, Spain \\ ${ }^{3}$ Hematology Department, University Hospital of Fuenlabrada, Madrid, Spain \\ ${ }^{4}$ Hematology Department, University Hospital Joan XXIII, Tarragona, Spain \\ ${ }^{5}$ Hematology Department, Infanta Leonor Hospital, Madrid, Spain \\ ${ }^{6}$ Hematology Department, Hospital of Segovia, Segovia, Spain \\ 7 Hematology Department, University Hospital Morales Meseguer, Murcia, Spain \\ ${ }^{8}$ Hematology Department, Hospital of León, León, Spain \\ ${ }^{9}$ Hematology Department, University Hospital of Santiago, Santiago de Compostela, Spain \\ ${ }^{10}$ Hematology Department, University Hospital Miguel Servet, Zaragoza, Spain \\ 11 Hematology Department, General Yagüe Hospital, Burgos, Spain \\ 12 Hematology Department and IBSAL, University Hospital of Salamanca, Salamanca, Spain
}

Correspondence to: Alberto Orfao, email: orfao@usal.es

Keywords: blastic plasmacytoid dendritic cell neoplasm, central nervous system, intrathecal prophylaxis, ALL therapy, flow cytometry

Received: January 12,2016 Accepted: January 21, 2016 Published: January 31, 2016

\section{ABSTRACT}

Blastic plasmacytoid dendritic cell neoplasm (BPDCN) is a rare aggressive myeloid neoplasm which shows a high rate of central nervous system (CNS) recurrence and overall survival (OS) of $<1$ year. Despite this, screening for CNS involvement is not routinely performed at diagnosis and intrathecal (IT) prophylaxis is not regularly administered in BPDCN. Here, we prospectively evaluated 13 consecutive BPDCN patients for the presence of CNS involvement by flow cytometry. Despite none of the patients presented with neurological symptoms, occult CNS involvement was detected in $6 / 10$ cases evaluated at diagnosis and $3 / 3$ studied at relapse/progression. BPDCN patients evaluated at diagnosis received IT treatment -either CNS prophylaxis $(n=$ 4 ) or active therapy $(n=6)$ - and all but one remain alive (median follow-up of 20 months). In contrast, all three patients assessed at relapse/progression died. The potential benefit of IT treatment administered early at diagnosis on OS and CNS recurrence-free survival of BPDCN was further confirmed in a retrospective cohort of another 23 BPDCN patients. Our results show that BPDCN patients studied at diagnosis frequently display occult CNS involvement; moreover, they also indicate that treatment of occult CNS disease might lead to a dramatically improved outcome of BPDCN. 


\section{INTRODUCTION}

Blastic plasmacytoid dendritic cell neoplasm $(\mathrm{BPDCN})$ is a rare disease -i.e. $<1 \%$ acute myeloblastic leukemia cases (AML)- with a historically dismal prognosis. Despite most BPDCN patients treated with different intensive chemotherapy regimens (e.g. CHOP, AML-type and acute lymphoblastic leukemia (ALL)-type regimens) achieve complete remission (CR), the great majority relapse early after $\mathrm{CR}$, with further resistance to therapy and a fatal outcome. Of note, a significant percentage $(\approx 30 \%)$ [1-3] of BPDCN relapses occur in the central nervous system (CNS), either as isolated CNS relapses or in the context of a systemic relapse. This is in line with the relatively high rate $(\approx 10 \%$ of cases) [1-6] of overt CNS involvement (i.e. confirmed by cytology) observed at diagnosis in patients with leukemic variant of BPDCN presenting with neurological symptoms.

Although BPDCN prognosis is extremely poor, patients treated with ALL-type regimens that include CNS prophylaxis [1-3] and/or allogeneic hematopoietic stem cell transplantation (AHSCT) [7, 8] appear to show a better outcome. Altogether, these findings suggest that occult CNS involvement might be already present at diagnosis in a significant fraction of BPDCN patients, and could contribute to explain the relatively high number of CNS recurrences [9].

Here, we prospectively evaluated for the first time the presence of CNS involvement by next generation flow cytometry (NGF) in 13 consecutive BPDCN patients studied at diagnosis $(n=10)$ or at disease recurrence $(n$ $=3$ ). The impact of CNS involvement and CNS-directed therapy in patient outcome was further validated in a retrospective series of 23 additional BPDCN patients.

\section{RESULTS AND DISCUSSION}

At diagnosis, most prospectively analyzed patients showed skin lesions $(n=10 / 13 ; 77 \%)$, peripheral blood $(7 / 13 ; 54 \%)$ and/or bone marrow $(9 / 13 ; 69 \%)$ involvement with normal neurological physical examination $(n=13 / 13$; $100 \%$ ) and a good performance status $(\mathrm{ECOG} \leq 0 / 1$ : $\mathrm{n}=12 / 13 ; 92 \%$ ) (Table 1). Despite this, cerebrospinal fluid (CSF) samples positive for tumor plasmacytoid dendritic cells were found in $6 / 10(60 \%)$ cases studied at diagnosis -median of 11 tumor cells/ $\mu 1$ (range: 0.6-47), representing $82 \%$ of the total CSF cellularity (range: $68 \%-95 \%$ )- in association with $\geq 20 \%$ bone marrow (BM) infiltration by tumor cells (6/6 vs. $1 / 4$ cases, respectively; $p=.03$ ). Except for the high rate of occult CNS involvement at diagnosis, prospectively analyzed here for the first time, the above described pattern of extranodal disease involvement has been previously reported as typical for BPDCN patients $[2,4,5]$.

Regarding the 3 patients referred at relapse (5, 7 and 14 months after diagnosis), none had been screened for
CNS disease (nor received CNS therapy) at diagnosis; all 3 had their CSF referred because of the onset of neurological symptoms, consisting of seizure in two patients and the presence of symptoms and signs of intracranial hypertension together with involvement of cranial nerves in the remaining case. They all showed CNS involvement by NGF with $>50 \%$ tumor cells $(958,5$ and 46 tumor cells $/ \mu 1$, respectively). These results confirm previous observations about the relatively high rate of overt CNS involvement in BPDCN, particularly at relapse [1-3]; in addition, these results also support the notion that many BPDCN patients may already have occult CNS disease at diagnosis, in the absence of neurological symptoms.

Despite BPDCN patients have been found to show already at diagnosis, a rather high rate $(\approx 10 \%)$ [1-6] of CNS involvement by conventional CSF cytology (e.g. similar to ALL patients), they are neither routinely screened for CNS involvement, nor receive CNS-directed therapy. This is even more striking because CNS relapses have been recurrently reported among BPDCN patients at relapse at higher frequencies than at diagnosis $(\approx 30 \%)$ [1-3]. Among other reasons, this might be due to the rarity of the disease -and hence the limited experience with these patients-, as well as the inclusion of BPDCN as a myeloid malignancy in the WHO classification [10], where CNS involvement at diagnosis is uncommon ( $<5 \%$ of patients) [11] and therefore, systematic CSF screening is not performed at diagnosis, in the absence of neurological symptoms. Moreover, NGF has not been routinely applied for the diagnostic screening of CNS involvement in BPDCN patients, despite its higher sensitivity over conventional cytology for detecting tumor cells on stabilized CSF samples $[12,13]$, and the fact that NGF can detect CNS disease before clinical symptoms emerge [14].

Of note, follow-up CSF samples obtained after triple intrathecal therapy (TIT) showed absence of tumor cells in $6 / 6 \mathrm{CSF}^{+}$cases studied at diagnosis, either after one $-5 / 6$ cases ( $83 \%$ )- or 4 doses of therapy (6/6 cases; Table 1). At the moment of closing this study, $5 / 6$ cases remained CNS-disease free, in continuous CR; the remaining patient (case\#4 in Table 1) had an isolated CNS relapse 6 months after diagnosis. Upon relapse, the patient received intrathecal (IT) chemotherapy, a single dose being sufficient to re-induce CR with NGF-negative CSF; subsequently, he underwent an AHSCT and remains disease-free for $>2$ years. At the moment of closing this study, only $1 / 10$ patients studied at diagnosis had died in CR (case \#5 in Table 1), the other 9 patients remaining alive in $\mathrm{CR}$ after a median follow-up of 20 months (range: 6-48 months). In contrast, those three patients studied at disease recurrence suffered disease progression and death, despite IT therapy was administered in $2 / 3$ cases (median overall survival (OS): 7 months) (Table 1). Overall, these findings suggest that BPDCN patients with occult CNS involvement at diagnosis might specifically benefit from 
Table 1: BPDCN patients included in the prospective cohort $(n=13)$ : Disease features at diagnosis and follow-up including patient outcome

\begin{tabular}{|c|c|c|c|c|c|c|c|c|c|c|c|c|c|c|c|c|c|c|c|c|c|c|c|c|c|}
\hline $\begin{array}{c}\text { Patient } \\
\text { code }\end{array}$ & Gender & Age & $\begin{array}{l}E C \\
O G \\
\end{array}$ & $\begin{array}{c}\text { Skin } \\
\text { lesions }\end{array}$ & $\begin{array}{l}\text { BM \% of } \\
\text { blast cells } \\
\text { at } \\
\text { diagnosis }\end{array}$ & $\begin{array}{c}\text { PB \% of } \\
\text { blast cells } \\
\text { at } \\
\text { diagnosis }\end{array}$ & $\begin{array}{c}\text { CSF } \\
\text { involvement } \\
\text { at diagnosis }\end{array}$ & $\begin{array}{c}\text { N. of blast } \\
\text { calls/1/11 } \\
\text { detected by } \\
\text { NGG in } \\
\text { CSF }\end{array}$ & $\begin{array}{c}\mathrm{CC} \\
\text { resultin } \\
\mathrm{CSF}\end{array}$ & $\begin{array}{l}\text { Systemic } \\
\text { chemo- } \\
\text { therapy }\end{array}$ & $\begin{array}{l}\text { IT therapy } \\
\text { at } \\
\text { diagnosis }\end{array}$ & $\begin{array}{c}\text { Response } \\
\text { to chemo- } \\
\text { therapy } \\
\end{array}$ & Relapse & $\begin{array}{c}\text { Sile of } \\
\text { relapse }\end{array}$ & $\begin{array}{c}\text { Time } \\
\text { (months) to } \\
\text { relappel } \\
\text { progression }\end{array}$ & $\begin{array}{c}\text { Positive } \\
\text { CSF" }\end{array}$ & $\begin{array}{c}\text { Time from } \\
\text { oliagnosis } \\
\text { tols } \\
\text { therpy } \\
\text { (days) } \\
\end{array}$ & $\begin{array}{c}\text { Type of IT } \\
\text { therapy }\end{array}$ & $\begin{array}{l}\text { N. of } T \\
\text { doses for } \\
\text { CSF } \\
\text { clearence }\end{array}$ & $\begin{array}{c}\text { CNS } \\
\text { relapsel } \\
\text { progression }\end{array}$ & AHSCT & $\begin{array}{c}\text { Disease } \\
\text { status }\end{array}$ & $\begin{array}{c}\text { Current } \\
\text { staus }\end{array}$ & $\begin{array}{l}\text { Cause } \\
\text { of } \\
\text { death }\end{array}$ & $\begin{array}{c}\text { Overall } \\
\text { survival } \\
\text { (months) }\end{array}$ \\
\hline \multicolumn{26}{|c|}{ NO CSF involvement at diagnosis by NGF ( $\mathrm{n}=4)$} \\
\hline \#3 & M & 60 & 1 & Yes & 29 & 4 & No & 0 & Negative & LAL-07FRAIL & Yes & $C R$ & No & & & No & 2 & $T \pi$ & NA & № & Yes & $C R$ & Alive & & 38 \\
\hline$\# 6$ & $F$ & 57 & 0 & Yes & 0 & 0 & No & 0 & NE & LAL-AR2011 & Yes & $C R$ & Yes & Cutaneous & 7 & No & 6 & TIT & NA & No & Yes & SD & Alive & & 27 \\
\hline \#9 & M & 67 & 0 & Yes & 0 & 0 & No & 0 & NE & LAL-07FRAIL & Yes & $C R$ & № & & & No & 29 & TIT & NA & № & No & $C R$ & Alive & & 20 \\
\hline$\# 11$ & M & 73 & 0 & Yes & 2 & 0 & No & 0 & NE & LAL-07FRAIL & Yes & $\mathrm{CR}$ & No & & & No & 14 & TाT & NA & No & No & $C R$ & Alive & & 9 \\
\hline Subtotal & $3 M / 1 F$ & $\begin{array}{c}64 \\
(57-73)\end{array}$ & 0 & $4 / 4$ & $\begin{array}{c}1 \\
(0-29)\end{array}$ & $\begin{array}{c}0 \\
(0-4)\end{array}$ & $0 / 4$ & 0 & 0/1 & & $4 / 4$ & $4 / 4 \mathrm{CR}$ & $1 / 4$ & & & $0 / 4$ & $\begin{array}{c}10 \\
(2-29)\end{array}$ & & NA & $0 / 4$ & $2 / 4$ & $3 / 4 \mathrm{CR}$ & $\begin{array}{c}4 / 4 \\
\text { Alive }\end{array}$ & & $\begin{array}{c}24 \\
(9-38)\end{array}$ \\
\hline \multicolumn{26}{|c|}{ CSF involvement at diagnosis by NGF $(n=6)$} \\
\hline$\# 1$ & M & 11 & NR & No & 80 & 83 & Yes & 5,1 & NE & LAL-AR/1993 & Yes & $C R$ & No & & & Yes & 11 & TT & 1 & № & No & CR & Alive & & 48 \\
\hline$\# 4$ & M & 16 & 0 & Yes & 94 & 68 & Yes & 0,6 & Negative & LAL-AR/2003 & Yes & $C R$ & Yes & Leptom & 5 & Yes & 2 & TIT \& Lip AraC & 1 & Yes & Yes & $C R$ & Alive & & 34 \\
\hline 45 & M & 67 & 0 & Yes & 80 & 45 & Yes & 2,2 & Positive & LAL-AR2011 & Yes & $C R$ & № & & & Yes & 3 & $T \pi$ & 1 & No & No & $C R$ & EXTUS & Sepsis & 2 \\
\hline$\# 10$ & м & 71 & 1 & yes & 88 & 0 & Yes & 6,4 & Negative & LAL-07FRAIL & Yes & $C R$ & No & & & res & 5 & TाI & 4 & No & No & $C R$ & Alive & & 15 \\
\hline$\# 12$ & $\mathrm{~F}$ & 79 & 2 & No & 68 & 4 & Yes & 46,6 & NE & LAL-07FRAIL & Yes & $C R$ & No & & & Yes & 7 & TIT \& Lip AraC & 1 & No & No & CR & Alive & & 7 \\
\hline$\# 13$ & M & 66 & 0 & Yes & 74 & 28 & Yes & 6,1 & NE & Hyper-CVAD & Yes & $C R$ & No & & & Yes & 6 & TIT & 1 & № & No & $C R$ & Alive & & 6 \\
\hline Subtotal & $5 M / 1 F$ & $\begin{array}{c}67 \\
(11-79)\end{array}$ & 0 & $4 / 6$ & $\begin{array}{c}80 \\
(68-94)\end{array}$ & $\begin{array}{c}37 \\
(0-83)\end{array}$ & $6 / 6$ & $\begin{array}{c}5.6 \\
(0.6-46.6)\end{array}$ & $1 / 3$ & & $6 / 6$ & 6/6 CR & $1 / 6$ & & & $6 / 6$ & $\begin{array}{c}6 \\
(2-11)\end{array}$ & & $\begin{array}{c}1 \\
(1-44)\end{array}$ & $1 / 6$ & $1 / 6$ & $6 / 6 \mathrm{CR}$ & $\begin{array}{l}5 / 6 \\
\text { Alive }\end{array}$ & & $\begin{array}{c}11 \\
(2-48)\end{array}$ \\
\hline \multicolumn{26}{|c|}{ CSF involvement at relapse/progression by NGF (not evaluated at diagnosis; $n=3$ ) } \\
\hline$\# 2$ & M & 58 & 0 & Yes & 0 & 0 & $\mathrm{NE}$ & 46,3 & NE & $\begin{array}{l}\text { Not riatments } \\
\text { CHOP at PD }\end{array}$ & No & PD & & & 14 & Yes & 426 & $\begin{array}{l}\text { MD MTXXarac } \\
\text { \& Lip AaraC }\end{array}$ & NC & Yes & No & PD & EXTUS & PD & 19 \\
\hline$\# 7$ & м & 78 & 1 & No & 94 & 33 & $\mathrm{NE}$ & 958,1 & $\mathrm{NE}$ & CYC\& PRED & № & PD & & & 5 & Yes & 173 & NA & NA & Yes & No & PD & EXTUS & PD & 5 \\
\hline \#8 & M & 75 & 0 & Yes & 0 & 0 & $\mathrm{NE}$ & 4,5 & Positive & FLUGAZA & № & SD & Yes & $\begin{array}{l}\text { BM, nodal } \\
\text { and leptom }\end{array}$ & 7 & Yes & 226 & Lip AraC & 1 & Yes & No & SD & EXITUS & PD & 7 \\
\hline Subtotal & 3M/0F & 75 & 0 & $2 / 3$ & 0 & 0 & $\mathrm{NE}$ & 46.3 & $1 / 1$ & & $0 / 3$ & $0 / 3 \mathrm{CR}$ & $1 / 1$ & & 7 & $3 / 3$ & 226 & & & $3 / 3$ & $0 / 3$ & $0 / 3 \mathrm{CR}$ & $\begin{array}{l}0 / 3 \\
\text { Alive }\end{array}$ & & 7 \\
\hline $\begin{array}{l}\text { Total } \\
n=13\end{array}$ & $\begin{array}{l}11 \mathrm{M} / \\
2 \mathrm{~F}\end{array}$ & $\begin{array}{c}67 \\
(11-79)\end{array}$ & 0 & $10 / 13$ & $\begin{array}{c}68 \\
(0-94)\end{array}$ & $\begin{array}{c}4 \\
(0-83)\end{array}$ & $6 / 10$ & $\begin{array}{c}6.1 \\
(0.6- \\
988.1) \\
\end{array}$ & $2 / 5$ & & 10/13 & 10/13 & $3 / 11$ & & $\begin{array}{c}7 \\
(5-14)\end{array}$ & $9 / 13$ & $\begin{array}{c}7 \\
(2-426)\end{array}$ & & 1 & $4 / 13$ & $3 / 13$ & $\begin{array}{c}9 / 13 \\
C R\end{array}$ & $\begin{array}{c}\text { 9/13 } \\
\text { Alive }\end{array}$ & & $\begin{array}{c}15 \\
(2-48)\end{array}$ \\
\hline \multicolumn{26}{|c|}{ Patients that received IT prophylaxis/therapy at diagnosis: Prospective vs validation cohort comparison } \\
\hline $\begin{array}{l}\text { Prospective } \\
(n=10)\end{array}$ & 8M/2F & 67 & & $8 / 10$ & 71 & 4 & 6,110 & 5.6 & $1 / 4$ & 10 ALL-type & 10/10 & 10110 CR & $2 / 10$ & $1 / 2$ CNS & 6 & $6 / 10$ & 6 & & & $1 / 10$ & $3 / 10$ & $9 / 10 \mathrm{CR}$ & 9/10 Alive & & 18 \\
\hline $\begin{array}{l}\text { Validation } \\
(n=5)\end{array}$ & 3M/2F & 31 & & $2 / 5$ & 73 & 0 & $2 / 2$ & NE & $2 / 2$ & 4/5 ALL-type & $5 / 5$ & $5 / 5 \mathrm{CR}$ & 215 & $2 / 2 \mathrm{NR}$ & 9 & $2 / 5$ & s15 & & & $0 / 4$ & $2 / 5$ & $3 / 5 \mathrm{CR}$ & 315 Aive & & 22 \\
\hline$P$ value & NS & 0.03 & & NS & NS & NS & NS & & & NS & NS & NS & NS & & NS & NS & 0.01 & & & NS & NS & NS & NS & & NS \\
\hline
\end{tabular}

ECOG: Eastern cooperative oncology group performance status; BM: Bone marrow; PB: Peripheral blood; CSF: Cerebrospinal fluid; NGF: Next generation flow cytometry; CC: Conventional cytology; IT: Intrathecal; CNS: Central nervous system; AHSCT: Allogeneic hematopoietic stem cell transplantation; M: Male; F: Female; NE: Not evaluated; PD: Progressive disease; CYC \& PRED: Cyclophosphamide and prednisone; PD: Progressive disease; CR: Complete remission; SD: Stable disease; Leptom: Leptomeningeal; TIT: Triple intrathecal therapy (methotrexate cytarabine and hydrocortisone); Lip: Liposomal; AraC: cytarabine; HD MTX: High dose methotrexate; NA: Not applicable; NC: No clearance of CSF observed; ALL: Acute lymphoblastic leukemia; NR: Not reported; NS: Not statistically significant. \#Due to the confirmed superiority of NGF versus CC in detecting occult leptomeningeal disease[12], and the limited volume of available CSF

sample, CC evaluation was not systematically performed in parallel *At any time (diagnosis or follow-up).
Patients were grouped according to their CSF status at diagnosis. Results in the summary groups are expressed as number of cases from all cases within the group (for categorical variables) or as median (for continuous variables) and minimum and maximum values.

All the patients had a normal neurological physical examination at diagnosis. 10 were screened for parenchymal involvement with a negative result in all these cases. All but case \#7 received IT treatment at some point of the disease but none CNS "LAdiotherapy" (NCT01358201, NCT00853008 and [21]) and Hyper-CVAD are ALL-type protocols, CHOP is a NHL-type protocol and FLUGAZA (NCT02319135) is an AML-type protocol. CYC \& PRED is a palliative regimen.

CNS-directed therapy, particularly if administered (early) at diagnosis; in line with this, early administration of TIT translated into a significantly better outcome and prolonged survival versus cases who did not receive CNS prophylaxis/therapy at diagnosis and presented with CNSrecurrent disease (Figure 1C and 1D).

In order to further validate our results, a series of 23 BPDCN patients previously reported in the literature [1] -and recruited in an earlier time period-, was retrospectively analyzed; of note, no major differences were observed between the two cohorts (prospectively $v s$. retrospectively analyzed patients) in terms of disease features and outcome (Table 1). Five of these 23 patients received IT prophylaxis at diagnosis and $4 / 23$ received CNS-directed therapy at relapse/progression (Table 2). In this independent validation cohort, patients who did receive IT prophylaxis at diagnosis also had prolonged CNS recurrence-free survival (CNS-RFS) $(p=.06)$ and OS $(p=.03)$ (Figure $1 \mathrm{E}$ and $1 \mathrm{~F}$ ). Univariate analysis of prognostic factors performed in the whole patient cohort $(\mathrm{n}=36)$ showed a favorable impact on CNS-RFS and/or OS for children ( $\mathrm{p}>.05 ; p=.03)$, patients receiving ALLtype therapy ( $p=.01 ; p=.002)$, AHSCT $(p>.05 ; p=.003)$ and IT prophylaxis/treatment at diagnosis $(p=.002 ; p<.001)$ (Figure $1 \mathrm{G}$ and $1 \mathrm{H}$ ), the later variable emerging as the only independent (favorable) prognostic factor for CNSRFS ( $\mathrm{p}=.02$, hazard ratio [HR] $=11.2,95 \%$ confidence interval $[\mathrm{CI}]: 1.4-88.8)$ and $\mathrm{OS}(p=.001, \mathrm{HR}=7.6,95 \%$ CI: $2.2-25.9)$.

The high rate of CNS involvement found in our study strongly suggests that the CNS could be a persistent blast-cell sanctuary in BPDCN patients with leukemic 

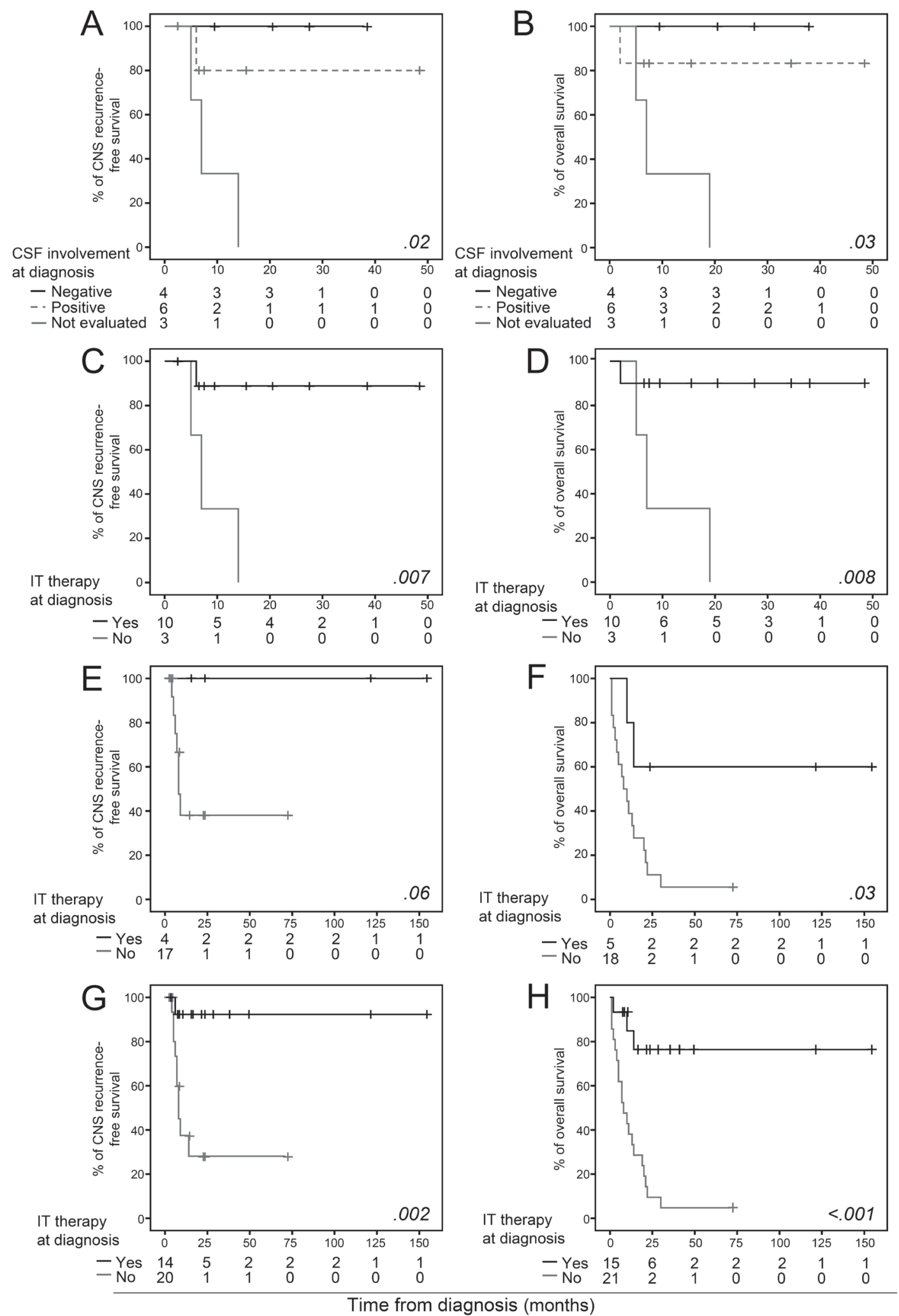

Figure 1: Prognostic impact of (occult) CSF involvement and administration of IT therapy at diagnosis in BPDCN patients. CNS-RFS (panels A, C, E and G) and OS (panels B, D, F and H) curves are separately shown for the prospective cohort (panels $\mathrm{A}, \mathrm{B}, \mathrm{C}$ and $\mathrm{D}$ ), the validation cohort (panels $\mathrm{E}$ and $\mathrm{F}$ ) and the combined (prospective and validation) cohort (panels $\mathrm{G}$ and $\mathrm{H}$ ). 
Table 2: BPDCN patients included in the validation cohort $(n=23)$ : Disease features at diagnosis and follow-up including patient outcome

\begin{tabular}{|c|c|c|c|c|c|c|c|c|c|c|c|c|c|c|c|c|c|c|c|}
\hline $\begin{array}{l}\text { Patient } \\
\text { code }\end{array}$ & Gender & Age & $\begin{array}{c}\text { Skin } \\
\text { lesions }\end{array}$ & $\begin{array}{l}\text { BM\% of } \\
\text { blast cells } \\
\text { at } \\
\text { diagnosis }\end{array}$ & $\begin{array}{l}\mathrm{PB} \% \text { of } \\
\text { blast cells } \\
\text { at } \\
\text { diagnosis }\end{array}$ & $\begin{array}{c}\text { CSF } \\
\text { involvement } \\
\text { at diagnosis }\end{array}$ & $\begin{array}{c}\text { Systemic } \\
\text { chemotherapy } \\
\text {-type regimen }\end{array}$ & $\begin{array}{l}\text { Response to } \\
\text { chemotherapy }\end{array}$ & Relapse & Site of relapse & $\begin{array}{c}\text { Time } \\
\text { (months) to } \\
\text { relapsel } \\
\text { progression }\end{array}$ & $\begin{array}{c}\text { Positive } \\
\text { CSF" }\end{array}$ & $\begin{array}{l}\text { Time from } \\
\text { diagnosis to } \\
\text { It therapy } \\
\text { (days) }\end{array}$ & CNS treatment & $\begin{array}{l}\text { CNS relapse/ } \\
\text { progression }\end{array}$ & AHSCT & Current status & $\begin{array}{c}\text { Cause of } \\
\text { death }\end{array}$ & $\begin{array}{l}\text { Overall } \\
\text { survival } \\
\text { (months) }\end{array}$ \\
\hline \multicolumn{20}{|c|}{ NO CNS IT prophylaxis administered at diagnosis $(n=18)$} \\
\hline$\# 4 R$ & M & 63 & No & 58 & 8 & NE & AML-type & $C R$ & Yes & CNS & 6 & Yes & 183 & TIT & Yes & No & EXITUS & PD & 10 \\
\hline \#6R & M & 76 & Yes & 0 & 0 & NE & NHL-type & $C R$ & Yes & Skin & 5 & No & & & No & No & EXITUS & PD & 13 \\
\hline$\# 8 R$ & M & 70 & Yes & 70 & 80 & NE & ALL-type & $\mathrm{CR}$ & Yes & CNS, BM & 8 & Yes & 244 & $\begin{array}{c}\begin{array}{c}\text { IIT \& } \\
\text { radiotherapy }\end{array} \\
\text { (n) }\end{array}$ & Yes & No & EXITUS & PD & 14 \\
\hline \#9R & M & 10 & Yes & 83 & 15 & NE & AML-type & $\mathrm{CR}$ & Yes & $\mathrm{NR}$ & 10 & No & & & NR & No & EXITUS & PD & 11 \\
\hline$\# 11 \mathrm{R}$ & $\mathrm{F}$ & 34 & No & 99 & 0 & NE & AML-type & $\mathrm{CR}$ & No & & & No & & & No & Yes & EXITUS & $\begin{array}{c}\text { AHSCT } \\
\text { complications }\end{array}$ & 7 \\
\hline$\# 19 R$ & M & 81 & Yes & 98 & 0 & NE & NHL-type & $C R$ & Yes & $\begin{array}{l}\text { CNS, skin and } \\
\text { lymph nodes }\end{array}$ & 7 & Yes & & & Yes & No & EXITUS & PD & 8 \\
\hline$\# 23 R$ & M & 81 & Yes & 75 & 0 & NE & ALL-type & ED & & & & No & & & No & No & EXITUS & Sepsis & 2 \\
\hline$\# 29 R$ & M & 78 & No & 65 & 35 & NE & AML-type & ED & & & & No & & & No & No & EXITUS & TreatTox & 1 \\
\hline$\# 31 R$ & M & 64 & Yes & 82 & 66 & NE & NHL-type & $C R$ & Yes & CNS, BM & 9 & Yes & 397 & $\begin{array}{l}\text { TIT \& Liposomal } \\
\text { AraC }\end{array}$ & Yes & No & EXITUS & PD & 20 \\
\hline$\# 33 R$ & M & 72 & Yes & 81 & 0 & NE & NHL-type & NR & & & 4 & Yes & & & Yes & No & EXITUS & PD & 4 \\
\hline$\# 38 R$ & M & 71 & Yes & 82 & 47 & NE & NHL-ype & ED & & & 3 & No & & & No & No & EXITUS & PD & 3 \\
\hline$\# 40 \mathrm{R}$ & M & 79 & Yes & 25 & 0 & NE & NHL-type & $C R$ & Yes & NR & 22 & No & & & No & No & EXITUS & PD & 22 \\
\hline$\# 42 R$ & M & 70 & Yes & 88 & 2 & NE & ALL-type & ED & & & & No & & & No & No & EXITUS & Sepsis & 1 \\
\hline$\# 58 R$ & $\mathrm{~F}$ & 54 & Yes & 60 & 40 & NE & AML-type & ED & & & & No & & & No & No & EXITUS & TreatTox & 1 \\
\hline \#59R & M & 46 & Yes & 90 & 10 & NE & NHL-type & $C R$ & Yes & CNS & 5 & Yes & & & Yes & No & EXITUS & PD & 5 \\
\hline$\# 61 R$ & M & 52 & No & 58 & 12 & NE & AML-type & $C R$ & No & & & No & & & No & Yes & Alive & & 71 \\
\hline$\# 63 R$ & M & 42 & No & 85 & 39 & NE & NHL-type & $C R$ & Yes & CNS, BM & 8 & Yes & 230 & & Yes & No & EXITUS & PD & 30 \\
\hline$\# 64 R$ & $\mathrm{~F}$ & 45 & No & 75 & 57 & NE & AML-type & $C R$ & No & & & No & & & No & No & EXITUS & Sepsis & 21 \\
\hline Subtotal & 15M/3F & $\begin{array}{c}67 \\
(10-81)\end{array}$ & $12 / 18$ & $\begin{array}{c}78 \\
(0-99)\end{array}$ & $\begin{array}{c}11 \\
(0-80)\end{array}$ & $18 \mathrm{NE}$ & $\begin{array}{c}3 / 18 \\
\text { ALL-type }\end{array}$ & $12 / 18 \mathrm{CR}$ & 9/12 & $6 / 9 \mathrm{CNS}$ & $\begin{array}{c}7 \\
(3-22)\end{array}$ & $7 / 18$ & $\begin{array}{c}237 \\
(183-397)\end{array}$ & & $7 / 17$ & $2 / 18$ & 1/18 Alive & & $\stackrel{9}{9}$ \\
\hline \multicolumn{20}{|c|}{ CNS IT prophylaxis administered at diagnosis $(n=5)$} \\
\hline$\# 12 R$ & $\mathrm{~F}$ & 31 & No & 45 & 0 & NE & ALL-type & $C R$ & No & & & No & $\leq 15$ & TIT & No & Yes & Alive & & 153 \\
\hline$\# 13 R$ & M & 12 & Yes & 73 & 0 & NE & ALL-type & $C R$ & No & & & No & $\leq 15$ & TIT & No & Yes & Alive & & 22 \\
\hline$\# 28 R$ & M & 53 & Yes & 76 & 0 & NE & ALL-type & $C R$ & Yes & NR & 10 & No & 15 & MTX \& AraC & No & No & EXITUS & PD & 14 \\
\hline \#35R & M & 8 & No & 85 & 0 & Yes & ALL-type & $C R$ & No & & & Yes & $\leq 15$ & MTX \& Dex & No & No & Alive & & 120 \\
\hline$\# 44 R$ & $\mathrm{~F}$ & 47 & No & 73 & 69 & Yes & AML-type & $C R$ & Yes & NR & 8 & Yes & 15 & Arac & NR & No & EXITUS & PD & 10 \\
\hline Subtotal & $3 \mathrm{M} / 2 \mathrm{~F}$ & $\begin{array}{c}31 \\
(8-53)\end{array}$ & $2 / 5$ & $\begin{array}{c}73 \\
(45-85)\end{array}$ & $\begin{array}{c}0 \\
(0-69)\end{array}$ & $2 / 2$ & 4/5 ALL-type & $5 / 5 \mathrm{CR}$ & $2 / 5$ & & $\begin{array}{c}9 \\
(8-10)\end{array}$ & $2 / 5$ & $\leq 15$ & & $0 / 4$ & $2 / 5$ & 3/5 Alive & & $\begin{array}{c}22 \\
(10-153)\end{array}$ \\
\hline$P$ value ${ }^{*}$ & NS & 0.02 & NS & NS & NS & & 0.02 & NS & NS & & NS & NS & 0.007 & & NS & NS & 0.02 & & 0.03 \\
\hline
\end{tabular}

BM: Bone marrow; PB: Peripheral blood; CSF: Cerebrospinal fluid; IT: Intrathecal; CNS: Central nervous Sytem; AHSCT:

Allogeneic hematopoietic stem cell transplantation; M: Male; F: Female; NE: Not evaluated; ALL: Acute Lymphoblastic Leukemia; NHL: Non-Hodgkin Lymphoma; AML: Acute Myeloblastic Leukemia; CR: Complete remission; ED: Early death; SD: Stable disease; NR: Not reported; TIT: Triple intrathecal therapy (Methotrexate cytarabine and hydrocortisone); AraC: Cytarabine; MTX: Metothrexate; Dex: Dexamethasone; PD: Progressive disease; TreatTox: Treatment toxicity; NS: Not statistically significant.

Results in the summary groups are expressed as number of cases from all cases within the group or as median. *At any time (diagnosis or follow-up) \#CNS IT prophylaxis administration at diagnosis versus not.

presentation, due to the limited power of cytostatic drugs to cross the blood-brain barrier into the CSF and brain parenchyma [15]. This reservoir of leukemic cells may also contribute to $\mathrm{BM} / \mathrm{systemic}$ disease recurrence. Longer survival rates observed in AHSCT BPDCN patients [7, 8], irrespective of IT medication administration, might be explained by the benefit of the antileukemic effect of donor allogeneic cells [16], able to circulate systemically and cross the blood-brain barrier. Unfortunately, AHSCT is not exempt from relapses $(\approx 1 / 3$ of patients) $[7,8]$, it cannot overcome the poor disease status beyond the first CR [7], and toxicity-related mortality is high [17]. Additionally, many patients are elderly or unfit subjects to undergo such an intensive therapeutic approach.

Recently, SL-401, a novel targeted therapy directed to the interleukin-3 receptor, has shown positive results on BPDCN patients with skin confined disease, whereas longterm benefit has not yet been observed in patients with the leukemic variant/phase of the disease [18]. No comparison can be established in terms of CNS relapses between both approaches (SL-401 vs. IT prophylaxis) since patients showing CNS involvement are excluded from the study (NCT02113982) and sites of relapse/progression after SL-401 administration have not been detailed $[18,19]$. Nonetheless, evaluation of IT injection of this targeted therapy will be of great interest, particularly in CNS relapses.

To the best of our knowledge, this is the first study reporting a strikingly high frequency of occult CNS involvement at diagnosis in BPDCN patients, and the potential benefit of CNS-directed therapy to reverse the poor outcome of BPDCN patients,. Indirectly, our results also suggest that occult CNS disease might contribute to explain the higher frequency of recurrences (e.g. CNS) observed in these patients, despite their high CR rates. Further prospective studies on larger BPDCN patient cohorts studied at diagnosis are necessary to confirm our findings. 


\section{MATERIALS AND METHODS}

\section{Ethics statement}

The study was approved by the Ethics Committee of the Cancer Research Center, and performed following the Declaration of Helsinki. Each participant gave his/her informed consent prior entering the study.

\section{Patients and samples}

Forty-one CSF samples from 13 consecutive BPDCN patients (11 males and 2 females; median age: 67 years, range: 11-79 years) were evaluated for the presence of CNS involvement by NGF. Cases were evaluated at diagnosis $(n=10)$ or at relapse/progression $(n=3)$ and subsequently, after IT therapy. Diagnosis of BPDCN was based on previously reported recommendations [10], including expression of markers claimed to be mandatory to allocate blast cells to the plasmacytoid dendritic cell lineage [20], by either flow cytometry or immunohistochemistry of peripheral blood specimens, BM samples or cutaneous lesions. The 10 patients studied at diagnosis received high-risk ALL-type treatment, based on the Spanish PETHEMA protocols (NCT01358201, NCT00853008 and [21])( $n=9)$ or HyperCVAD therapy $(n=1)$, including one dose of TIT as CNS prophylaxis at each treatment phase (Table 1). For CSF-positive cases, additional IT treatment was given -TIT every $72 \mathrm{~h}(n=$ 4) or liposomal cytarabine (50mg) every two weeks ( $n$ $=2$ )- until two consecutive CSF-negative samples were obtained. In turn, patients with recurrent disease $(n=3)$ received AML-type or NHL-type (CHOP) therapy (Table $1)$.

In order to validate the impact of CNS involvement and CNS-directed therapy on patient outcome, an independent validation cohort of $23 \mathrm{BPDCN}$ treated with ALL- $(n=7)$, AML- $(n=8)$ or NHL-type $(n=8)$ therapeutic regimens was retrospectively analyzed [1]. The intrathecal treatment administered to these patients is detailed in Table 2.

Multiparameter NGF immunophenotypic studies were performed on Transfix ${ }^{\mathrm{TM}_{\text {-stabilized }} \text { CSF samples }}$ (Cytomark, Buckingham, UK) following the EuroFlow panels and protocols $[12,22]$. Data analysis was performed with the Infinicyt software (Cytognos, Salamanca, Spain). The lower cut-off for CNS involvement was defined as a cluster $\geq 10$ events with the appropriate phenotype, based on a 10-parameter tube $(\geq 0.001$ cells $/ \mu \mathrm{l})$.

\section{Statistical analyses}

The Mann-Whitney U (for continuous variables) and the $\chi^{2}$ tests (for categorical variables) were used to determine the statistical significance of differences observed between groups (PASW 19 statistical software, IBM SPSS Statistics, IBM, Armonk, NY, USA). OS and CNS-RFS curves were plotted according to the method of Kaplan-Meier and compared using the (one-sided) logrank test. Those variables showing prognostic value in the univariate analysis were also evaluated by multivariate analysis using a Cox stepwise regression model. Statistically significance was set at $\mathrm{p}$ values $<.05$.

\section{ACKNOWLEDGMENTS}

The authors would like to thank Antonio López (University of Salamanca, Spain), Mary Luz Amigo (Hospital Morales Meseguer, Murcia, Spain), Belén Navarro (Hospital Puerta de Hierro, Madrid, Spain), Fernando Principe (Hospital Sao Joao, Oporto, Portugal) and Berta Valverde (Hospital Dr. Carlos Sáenz Herrera, San José, Costa Rica), for their contribution to this work.

\section{CONFLICTS OF INTEREST}

The authors declare that there are no conflicts of interest to disclose.

\section{GRANT SUPPORT}

This work was supported by grants RD06/0020/0035 and RD12/0036/0048 from RETICS (Instituto de Salud Carlos III, Ministerio de Economía y Competitividad, Madrid, Spain and Fondos FEDER) and grant EDU/878/2004 from Junta de Castilla y León and Fondo Social Europeo.

\section{Editorial note}

This paper has been accepted based in part on peerreview conducted by another journal and the authors' response and revisions as well as expedited peer-review in Oncotarget.

\section{REFERENCES}

1. Martin-Martin L, Lopez A, Vidriales B, Caballero MD, Rodrigues AS, Ferreira SI, Lima M, Almeida S, Valverde B, Martinez P, Ferrer A, Candeias J, Ruiz-Cabello F, Buadesa JM, Sempere A, Villamor N, et al. Classification and clinical behavior of blastic plasmacytoid dendritic cell neoplasms according to their maturation-associated immunophenotypic profile. Oncotarget. 2015; 6:19204- 
19216. doi: 10.18632/oncotarget.4146.

2. Feuillard J, Jacob MC, Valensi F, Maynadie M, Gressin R, Chaperot L, Arnoulet C, Brignole-Baudouin F, Drenou B, Duchayne E, Falkenrodt A, Garand R, Homolle E, Husson B, Kuhlein E, Le Calvez G, et al. Clinical and biologic features of CD4(+)CD56(+) malignancies. Blood. 2002; 99:1556-1563.

3. Pagano L, Valentini CG, Pulsoni A, Fisogni S, Carluccio P, Mannelli F, Lunghi M, Pica G, Onida F, Cattaneo C, Piccaluga PP, Di Bona E, Todisco E, Musto P, Spadea A, D'Arco A, et al. Blastic plasmacytoid dendritic cell neoplasm with leukemic presentation: an Italian multicenter study. Haematologica. 2013; 98:239-246.

4. Tsagarakis NJ, Kentrou NA, Papadimitriou KA, Pagoni M, Kokkini G, Papadaki H, Pappa V, Marinakis T, Anagnostopoulos NI, Vadikolia C, Anagnostopoulos A, Angelopoulou MK, Terpos E, Poziopoulos C, Anargyrou K, Rontogianni D, et al. Acute lymphoplasmacytoid dendritic cell (DC2) leukemia: results from the Hellenic Dendritic Cell Leukemia Study Group. Leuk Res. 2010; 34:438-446.

5. Hashikawa K, Niino D, Yasumoto S, Nakama T, Kiyasu J, Sato K, Kimura Y, Takeuchi M, Sugita Y, Hashimoto T and Ohshima K. Clinicopathological features and prognostic significance of CXCL12 in blastic plasmacytoid dendritic cell neoplasm. J Am Acad Dermatol. 2012; 66:278-291.

6. Pemmaraju N, Kantarjian HM, Cortes JE, Duvic M, Khoury JD, Patel K, Daver N, O’Brien S, Pierce S, Garcia-Manero G, Jabbour E, Jain N, Faderl S, Thomas D, Frankel AE, Qazilbash MH, et al. Blastic Plasmacytoid Dendritic Cell Neoplasm (BPDCN): A Large Single-Center Experience: Analysis of Clinical and Molecular Characteristics and Patient Outcomes. Blood. 2015; 126:3746-3746.

7. Aoki T, Suzuki R, Kuwatsuka Y, Kako S, Fujimoto K, Taguchi J, Kondo T, Ohata K, Ito T, Kamoda Y, Fukuda T, Ichinohe T, Takeuchi K, Izutsu K and Suzumiya J. Longterm survival following autologous and allogeneic stem cell transplantation for blastic plasmacytoid dendritic cell neoplasm. Blood. 2015; 125:3559-3562.

8. Roos-Weil D, Dietrich S, Boumendil A, Polge E, Bron D, Carreras E, Iriondo Atienza A, Arcese W, Beelen DW, Cornelissen JJ, Kroger N, Milone G, Rossi G, Jardin F, Peters C, Rocha V, et al. Stem cell transplantation can provide durable disease control in blastic plasmacytoid dendritic cell neoplasm: a retrospective study from the European Group for Blood and Marrow Transplantation. Blood. 2013; 121:440-446.

9. Jegalian AG, Buxbaum NP, Facchetti F, Raffeld M, Pittaluga S, Wayne AS and Jaffe ES. Blastic plasmacytoid dendritic cell neoplasm in children: diagnostic features and clinical implications. Haematologica. 2010; 95:1873-1879.

10. Facchetti F, Jones M and Petrella T. (2008). Blastic plasmacytoid dendritic cell neoplasm. In: Swerdlow SH CE, Harris NL, Jaffe ES, Pileri SA, Stein H et al., ed. WHO classification of tumors of hematopoietic and lymphoid tissues. (Lyon, France: International Agency for Research on Cancer (IARC)), pp. 145-147.

11. Rees JK, Gray RG, Swirsky D and Hayhoe FG. Principal results of the Medical Research Council's 8th acute myeloid leukaemia trial. Lancet. 1986; 2:1236-1241.

12. Quijano S, Lopez A, Manuel Sancho J, Panizo C, Deben G, Castilla C, Antonio Garcia-Vela J, Salar A, Alonso-Vence N, Gonzalez-Barca E, Penalver FJ, Plaza-Villa J, Morado M, Garcia-Marco J, Arias J, Briones J, et al. Identification of leptomeningeal disease in aggressive B-cell non-Hodgkin's lymphoma: improved sensitivity of flow cytometry. J Clin Oncol. 2009; 27:1462-1469.

13. Bromberg JE, Breems DA, Kraan J, Bikker G, van der Holt B, Smitt PS, van den Bent MJ, van't Veer M and Gratama JW. CSF flow cytometry greatly improves diagnostic accuracy in CNS hematologic malignancies. Neurology. 2007; 68:1674-1679.

14. Hegde U, Filie A, Little RF, Janik JE, Grant N, Steinberg SM, Dunleavy K, Jaffe ES, Abati A, Stetler-Stevenson M and Wilson WH. High incidence of occult leptomeningeal disease detected by flow cytometry in newly diagnosed aggressive B-cell lymphomas at risk for central nervous system involvement: the role of flow cytometry versus cytology. Blood. 2005; 105:496-502.

15. Pistoia V and Raffaghello L. Disclosing the mysteries of the central nervous system sanctuary for acute lymphoblastic leukemia cells. Leuk Res. 2011; 35:699-700.

16. Aoki J, Ishiyama K, Taniguchi S, Fukuda T, Ohashi K, Ogawa H, Kanamori H, Eto T, Iwato K, Sakamaki H, Morishima Y, Nagamura T, Atsuta Y and Takami A. Outcome of allogeneic hematopoietic stem cell transplantation for acute myeloid leukemia patients with central nervous system involvement. Biol Blood Marrow Transplant. 2014; 20:2029-2033.

17. Leclerc M, Peffault de Latour R, Michallet M, Blaise D, Chevallier P, Rohrlich P-S, Turlure P, Nguyen S, Jardin F, Yakoub-Agha I, Moukhtari L, Raus N and Maury S. Reduced Intensity Versus Myelo-Ablative Conditioning Regimen before Allogeneic Hematopoietic Stem Cell Transplantation for Blastic Plasmacytoid Dendritic Cell Neoplasm: A Retrospective Study of the French Society of Bone Marrow Transplantation and Cell Therapy (SFGMTC). Blood. 2015; 126:3208-3208.

18. Frankel AE, Woo JH, Ahn C, Pemmaraju N, Medeiros BC, Carraway HE, Frankfurt O, Forman SJ, Yang XA, Konopleva M, Garnache-Ottou F, Angelot-Delettre F, Brooks C, Szarek M and Rowinsky E. Activity of SL-401, a targeted therapy directed to interleukin-3 receptor, in blastic plasmacytoid dendritic cell neoplasm patients. Blood. 2014; 124:385-392.

19. Sweet KL, Pemmaraju N, Lane AA, Stein AS, Vasu S, Blum W, Rizzieri DA, Wang ES, Rowinsky EK, Szarek M, Brooks CL, Disalvatore S, Liu D, Duvic M, Schwartz JD and Konopleva M. Lead-in Stage Results of a Pivotal Trial of SL-401, an Interleukin-3 Receptor (IL-3R) Targeting Biologic, in Patients with Blastic Plasmacytoid Dendritic 
Cell Neoplasm (BPDCN) or Acute Myeloid Leukemia (AML). Blood. 2015; 126:3795-3795.

20. Garnache-Ottou F, Feuillard J, Ferrand C, Biichle S, Trimoreau F, Seilles E, Salaun V, Garand R, Lepelley P, Maynadie M, Kuhlein E, Deconinck E, Daliphard S, Chaperot L, Beseggio L, Foisseaud V, et al. Extended diagnostic criteria for plasmacytoid dendritic cell leukaemia. British journal of haematology. 2009; 145:624636.

21. Ribera JM, Ortega JJ, Oriol A, Bastida P, Calvo C, Perez-Hurtado JM, Gonzalez-Valentin ME, MartinReina V, Molines A, Ortega-Rivas F, Moreno MJ, Rivas C, Egurbide I, Heras I, Poderos C, Martinez-Revuelta E, et al. Comparison of intensive chemotherapy, allogeneic, or autologous stem-cell transplantation as postremission treatment for children with very high risk acute lymphoblastic leukemia: PETHEMA ALL-93 Trial. J Clin Oncol. 2007; 25:16-24.

22. van Dongen JJ, Lhermitte L, Bottcher S, Almeida J, van der Velden VH, Flores-Montero J, Rawstron A, Asnafi V, Lecrevisse Q, Lucio P, Mejstrikova E, Szczepanski T, Kalina T, de Tute R, Bruggemann M, Sedek L, et al. EuroFlow antibody panels for standardized n-dimensional flow cytometric immunophenotyping of normal, reactive and malignant leukocytes. Leukemia. 2012; 26:1908-1975. 